\title{
Terminal 3-fold Divisorial Contractions of a Surface to a Curve I
}

\author{
NIKOLAOS TZIOLAS \\ Department of Mathematics, University of Crete, Knossos Ave., Heraklion 71409, Greece. \\ e-mail:tziolas@math.uch.gr
}

(Received: 6 November 2001; accepted: 3 July 2002)

\begin{abstract}
Let $\Gamma \subset X$ be a smooth curve on a 3-fold which has only index 1 terminal singularities along $\Gamma$. In this paper we investigate the existence of extremal terminal divisorial contractions $E \subset Y \longrightarrow \Gamma \subset X$, contracting an irreducible surface $E$ to $\Gamma$. We consider cases with respect to the singularities of the general hypersurface section $S$ of $X$ through $\Gamma$. We completely classify the cases when $S$ is $A_{i}, i \leqslant 3$, and $D_{2 n}$ for any $n$.
\end{abstract}

Mathematics Subject Classifications (2000). Primary: 14E30, 14E35.

Key words. algebraic geometry.

\section{Introduction}

One of the main objectives of birational geometry is to identify in each birational class of varieties some distinguished members which are 'simple' and are called minimal models, and then study the structure of birational maps between them. In dimension two, satisfactory results were known for over one hundred years. In higher dimensions, the minimal model program (MMP) was developed to search for minimal models. After contributions of Reid, Mori, Kawamata, Kollár, Shokurov and others, the program was completed in dimension three by Mori in 1988. A projective variety $X$ is called a minimal model iff it is Q-factorial, terminal and $K_{X}$ is nef. According to Mori's theorem, for any Q-factorial, terminal projective 3 -fold $X$, there is a sequence of birational maps $X \longrightarrow X^{\prime}$, such that $X^{\prime}$ is either a minimal model or has the structure of a Mori fiber space. The birational maps that appear are divisorial contractions and flips. Any birational map between minimal models is an isomorphism in codimension one and a composition of flops [Ko-Mo98]. Terminal flops were classified by the work of Kollár [Ko91].

The structure of birational maps between Fano fiber spaces is complicated. The Sarkisov program was developed by Sarkisov, Reid and Corti to factorize birational maps between these spaces as a composition of 'elementary links' [Cor95]. These links consist of flops, flips and divisorial contractions. Therefore to understand the structure of birational maps between Fano fiber spaces, it is important to understand divisorial contractions and flips. Flips were classified by Kollár and Mori [Ko-Mo92]. The structure of divisorial contractions is still an open problem. 
Let $E \subset Y \stackrel{f}{\longrightarrow} \Gamma \subset X$ be a divisorial contraction. Mori and Cutkoski completely classify such contractions when $Y$ is Gorenstein. In particular, if $\operatorname{dim} \Gamma=1$, then $X$ is smooth along $\Gamma$ and $Y$ is just the blow up of $X$ along $\Gamma$. Kawamata [Kaw94] showed that if there is a point $P \in \Gamma \subset X$ such that $P \in X$ is a cyclic quotient terminal singularity, then $\Gamma=\{P\}$ and $f$ is a weighted blow up. Divisorial contractions of a surface to a point, i.e. when $\Gamma=\{p t\}$, have been studied by Luo, Corti, Kawakita and others.

This paper studies divisorial contractions of a surface to a curve, i.e. when $\operatorname{dim} \Gamma=1$ and $X$ has only index 1 terminal singularities along $\Gamma$. It is not always true that given $\Gamma \subset X$, then there is a terminal contraction of a surface to $\Gamma$. We investigate when there is one, give criteria for existence or not and in the case that there is a terminal contraction we also describe the singularities of $Y$.

The natural setting of the problem is to replace $X$ with an analytic neighborhood of a singular point $P \in \Gamma$ and study the existence of divisorial extremal neighborhoods over the germ $P \in X$.

By [Ko-Mo92], if a terminal divisorial extremal neighborhood with irreducible central curve exists then there is a DuVal section $\Gamma \subset S \subset X$. In this paper we will assume the existence of a DuVal section $S$ of $X$ through $\Gamma$ and we base the classification of contractions on the type of singularities the general $S$ as above has, instead of $X$ itself. Theorem 1.6 shows that under certain conditions there is always a canonical contraction. The objective of the rest of this paper is to investigate when the contraction is terminal in the case that $\Gamma$ is smooth. This smoothness condition on $\Gamma$ is not a big restriction for applications to the Sarkisov program. In particular, to study the birational rigidity of a Fano 3-fold, it is important to exclude certain curves as maximal centers. In most cases [Cor-Rei00] these are either lines or conics.

In order to investigate the existence of a terminal contraction, it is important to obtain normal forms for the equations of $\Gamma \subset S \subset X$. This is done in Proposition 4.7. Theorem 5.1 gives criteria for existence in the case that $S$ is $A_{i}$ with $i \leqslant 3$, and Theorem 6.1 treats the case that $S$ is $D_{2 n}$.

There is an important difference between the $D_{2 n}$ and $D_{2 n+1}$, as well as for the higher $A_{n}$ cases. The main difficulty is the explicit calculation of the Q-factorialization $Z$ of $E_{1}$, as appears in the proof of Theorem 1.6. The reason of this difficulty becomes clear in Lemma 6.2.

\section{Uniqueness and Canonical Contractions}

DEFINITION 1.1. A 3-fold divisorial contraction is a morphism $f: E \subset Y \longrightarrow$ $\Gamma \subset X$, such that $X$ and $Y$ are Q-factorial, $Y-E \cong X-\Gamma$ and $E$ is a prime divisor, $-K_{Y}$ is $f$-ample, and $\rho(Y / X)=1$.

Under certain conditions, contractions of a surface to a curve are unique, as shown by the next proposition. In particular, this is the case for terminal contractions. 
PROPOSITION 1.2 ([Ko-Mo98]). Let $f: E \subset Y \longrightarrow \Gamma \subset X$ be a 3-fold divisorial contraction of an irreducible surface $E$ to a curve $\Gamma$. Suppose that $X, Y$ are normal, $\operatorname{dim} f\left(Y^{\mathrm{sing}}\right)=0, X$ has isolated singularities and $-E$ is $f$-ample. Then

$$
Y \cong \operatorname{Proj} \bigoplus_{d \geqslant 0} \mathcal{I}_{\Gamma, X}^{(d)}
$$

However this is not true if the condition $\operatorname{dim} f\left(Y^{\text {sing }}\right)=0$ is removed, as shown by the next example:

EXAMPLE 1.3. Let $X$ be given by $x^{2}+y^{2} z+z^{3}+t^{5}=0$ and $\Gamma: x=z=t=0$. Then there are 2 nonisomorphic canonical divisorial contractions $g_{i}: S_{i} \subset Z_{i} \longrightarrow$ $\Gamma \subset X$ contracting the surfaces $S_{i} \subset Z_{i}$ to $\Gamma . Z_{1}$ has index 1 and is singular along a section of $S_{1} \longrightarrow \Gamma$ and $Z_{2}$ has index 2 and its singular locus is $g_{2}^{-1}(0)$.

Proof. The statement about the contraction $Z_{2} \longrightarrow X$ follows from Theorem 6.1. So here we will only show how to construct the contraction $g_{1}: Z_{1} \longrightarrow X$. Let $I=\left(x^{2}, z, t\right)$. Let $g_{1}: Z_{1}=B_{I} X \longrightarrow X$ be the blow up of the ideal $I$ in $X$. $Z_{1} \subset \mathbb{C}^{4} \times \mathbb{P}^{2}$. Let $u, v, w$ be coordinates for $\mathbb{P}^{2}$. Look at the chart $w \neq 0$. $Z_{1}$ is given by

$$
\begin{aligned}
& x^{2}-u t=0 \\
& u+y^{2} v+v^{3} t^{2}+t^{4}=0
\end{aligned}
$$

There is only one $g_{1}$-exceptional divisor $S_{1}$ given by $x=t=u+y^{2} u=0 . Z_{1}$ is easily seen to be singular along the line $l: x=t=u=v=0$ which lies over $\Gamma$. Moreover, since $Z_{1}$ is a complete intersection, it has index 1 .

The existence of a divisorial contraction as above, is equivalent to the finite generation of $\bigoplus_{d \geqslant 0} \mathcal{I}_{\Gamma, X}^{(d)}$. This is local around any singular point of $X$ on $\Gamma$. Therefore the proper way to study this problem is to replace $X$ with an analytic neighborhood of a singular point $P \in \Gamma$.

The singularities of the general hyperplane section $S$ of $X$ through $\Gamma$ are very important for the study of terminal contractions as shown by the following theorem.

THEOREM 1.4 ([Ko-Mo92]). Let $f: Y \supset C \longrightarrow X \ni P$ be an extremal neighborhood with irreducible central curve $C$. Then the general member $E_{Y}$ of $\left|-K_{Y}\right|$ and $E_{X}=f\left(E_{Y}\right) \in$ $\left|-K_{X}\right|$ have only DuVal singularities. Moreover, the minimal resolution of $E_{X}$ dominates $E_{Y}$.

If the germ $P \in X$ is Q-factorial, then the central curve is irreducible and the above theorem applies. Moreover, Professor S. Mori told me that he has recently 
proved the above theorem when the central curve is reducible and the contraction is flipping. However, he did not check the divisorial case yet but he feels that his method may prove this case as well. Therefore in order to study and classify divisorial extremal neighborhoods it is not really restrictive to assume the existence of a DuVal section containing the curve. Our problem is thus reduced to the following:

QUESTION-DEFINITION 1. Let $(P \in X)$ be the germ of an index 13 -fold terminal singularity and $P \in \Gamma \subset X$ a curve. Assume that there exist a DuVal section $S$ of $X$ containing $\Gamma$. Does there exist a projective birational morphism $f: Y \longrightarrow X$ from a variety $Y$ with only terminal singularities whose exceptional set $E$ is a Q-Cartier irreducible divisor such that $-E$ is $f$-ample and is mapped onto $\Gamma$. We will call any such contraction a divisorial contraction of type I.

By this replacement, the Q-factoriality of $X$ is lost as well as the $\rho(Y / X)=1$ requirement. However, by [Art69] and [Art70], $P \in X$ is algebraic and we may also work with an algebraic neighborhood of $P \in X$ that is Q-factorial, if we need to.

From Theorem 1.4 and the above discussion it follows that we expect that if a terminal contraction exists, then there is a DuVal section $S$ containing $\Gamma$. The converse is not true as shown by Example 5.3.

The next example shows that there are cases when there is no DuVal section containing $\Gamma$.

EXAMPLE 1.5. Let $X$ be given by $x^{2}+y^{3}+z^{3}+y t^{6}=0$ and $\Gamma: x=y=z=0$. Then there is no DuVal section of $X$ containing $\Gamma$. Moreover, the blow up $Y=B_{\Gamma} X$ of $X$ along $\Gamma$ is not even canonical (this shows that the conditions of Proposition 2.6 are needed).

Proof. First observe that $0 \in X$ is a $c D_{4}$ singularity and therefore terminal. The section $t=0$ is the surface $x^{2}+y^{3}+z^{3}=0$, which is easily seen to be a $D_{4}$ singularity. A general hyperplane containing $\Gamma$ in $\mathbb{C}^{4}$ is given by $x=a y+b z$. Hence the corresponding section of $X$ is $S:(a y+b z)^{2}+y^{3}+z^{3}+y t^{6}=0$. It is not difficult to check that after two blow ups it becomes nonnormal and hence it cannot be DuVal. Hence there is no DuVal section of $X$ containing $\Gamma$.

Now let $f: Y=B_{\Gamma} X \longrightarrow X$ be the blow up of $X$ along $\Gamma$. In the affine chart $x=x z$, $y=y z, Y$ is given by $x^{2} z+y^{3} z^{2}+z^{2}+y t^{6}=0$. Moreover,

$$
x^{2} z+y^{3} z^{2}+z^{2}+y t^{6}=x^{2} z+\left(y^{3}+1\right) z^{2}+y t^{6} \sim x^{2} z+z^{2}+y t^{6}
$$

which is singular along the line $l: x=z=t=0$. A typical section given by $y=a$ is $x^{2} z+z^{2}+a t^{6}=0$. Blow up the origin twice as before to get a nonnormal surface. Hence $Y$ has a line of at best $\log$ canonical singularities.

Hence the existence of a DuVal section through $\Gamma$ is important to conclude that $Y=B_{\Gamma} X$ is canonical in Proposition 2.6. 
The previous example shows the significance of the existence of a DuVal section $S$ of $X$ through $\Gamma$. Therefore from now on we will assume the existence of such a section. In fact, we will study the existence of terminal contractions by considering cases with respect to the type of singularities of the general $S$ through $\Gamma$, instead of the singularities of $X$ itself. To start with we will show that there is always a canonical contraction.

THEOREM 1.6. Let $P \in X$ be the germ of an index 1 terminal 3-fold singularity and $P \in \Gamma \subset X$ an irreducible curve having at worst lci singularities. Suppose there is a DuVal section $S$ of $X$ containing $\Gamma$. Then there is a divisorial contraction of type $I$ $g: E \subset W \longrightarrow \Gamma \subset X$ contracting an irreducible surface $E$ to $\Gamma$, with $W$ canonical and $\operatorname{dim} g\left(W^{\text {sing }}\right)=0$. In particular, $R(\Gamma, X)=\bigoplus_{n \geqslant 0} \mathcal{I}_{\Gamma, X}^{(n)}$ is finitely generated.

The proof of the previous theorem will be given in Section 3 .

\section{Some Easy Lemmas}

DEFINITION 2.1. Let $X$ be a normal variety, and $D$ a Q-Cartier divisor in $X$. Let $P \in X$. Then the index of $D$ at $P, \operatorname{index}_{P}(D)$, is defined to be the smallest $r \in \mathbb{N}-0$ such that $r D$ is Cartier at $P$. The global index of $D$ in $X$, $\operatorname{index}_{X}(D)$ is the smallest $r \in \mathbb{N}-0$ such that $r D$ is Cartier.

LEMMA 2.2. Let $X$ be a threefold. Suppose that its singular locus is an irreducible curve $\Gamma$ and that it has only hypersurface singularities. Let $D$ be a Q-Cartier divisor on $X$. Then

$$
\operatorname{index}_{P}(D)=\operatorname{index}_{X}(D)
$$

for any point $P \in \Gamma$. Hence the index of $D$ can be computed at any point of $\Gamma$.

Proof. The proof is almost identical to that for the case of isolated index 1 terminal singularities that appears in [Kaw88]. I am not aware of a reference for this more general case and therefore I include it for the convenience of the reader.

Let $r=\operatorname{index}_{X}(D)$. Then there are finitely many possibilities for $\operatorname{index}_{P}(D)$. Suppose that $r_{1} \leqslant r_{2} \leqslant \cdots \leqslant r_{k}=r$ be these possibilities. Suppose that $r_{1}=\operatorname{index}_{P_{1}}(D)$. Since $\Gamma$ is irreducible, $r_{1} D$ is Cartier at all but finitely many points where $D$ has index greater than $r_{1}$. We can assume that it is only one, say $P$, since the result is local. Hence $\operatorname{index}_{P}(D)=r$. Then $D^{\prime}=r_{1} D$ is Cartier everywhere except $P$. Let $\pi: W \longrightarrow X$ be the index 1 cover of $D^{\prime}$. Hence $W-\pi^{-1}(P) \longrightarrow X-P$ is étale. $X$ has hypersurface singularities and therefore by [Mil68, Theorem 5.2] $\pi_{1}(X-P)=0$. Hence the cover is trivial and therefore $D^{\prime}$ is Cartier.

LEMMA 2.3. Let $P \in \Gamma \subset X$. Suppose that $P \in X$ is a three-dimensional index 1 terminal singularity, and that $P \in \Gamma$ is a plane curve singularity. Let $f: Y=B_{\Gamma} X \longrightarrow X$ be 
the blow up of $X$ along $\Gamma$. Then $f^{-1}(\Gamma)=E_{1}+m E_{2}$. $E_{1}$ is an irreducible surface over $\Gamma$. If $P \in \Gamma$ is smooth, then $E_{2} \cong \mathbb{P}^{2}$. Otherwise $E_{2} \cong \mathbb{P}^{2}$ or $E_{2}=\emptyset$.

Proof. The result is local around $P$. Since $P \in X$ is an index 1 terminal singularity, $P$ is a cDV point. Hence, we can assume that $X \subset \mathbb{C}^{4}$. Suppose $P \in \Gamma$ is smooth. First we will show that $\operatorname{dim} f^{-1}(P)=2$. Suppose not. Let $P \in S \subset X$ be a general hypersurface section transversal to $\Gamma$. Then $P \in S$ is DuVal and $S^{\prime}=f_{*}^{-1} S$ is just the blow up of $P$ in $S$. In particular, it is normal and $K_{S^{\prime}}=f^{*} K_{S}$. If $\operatorname{dim} f^{-1}(P)=1$, then $f^{*} S=S^{\prime}$. In particular, $S^{\prime}$ is Cartier. Moreover, since $f$ is generically the blow up of a smooth curve, $K_{Y}=f^{*} K_{X}+E_{1}$. Since $Y$ is CM and $S^{\prime}$ Cartier,

$$
\begin{aligned}
K_{S^{\prime}} & =\left.\left(K_{Y}+S^{\prime}\right)\right|_{S^{\prime}}=\left.\left(f^{*} K_{X}+E_{1}+f^{*} S\right)\right|_{S^{\prime}} \\
& =\left.\left(f^{*}\left(K_{X}+S\right)+E_{1}\right)\right|_{S^{\prime}}=f^{*} K_{S}+\left.E_{1}\right|_{S^{\prime}}=K_{S^{\prime}}+\left.E_{1}\right|_{S^{\prime}} .
\end{aligned}
$$

It now follows that $\left.E_{1}\right|_{S^{\prime}}=0$, which is impossible. Hence, $\operatorname{dim} f^{-1}(P)=2$. Now in both cases, since $\Gamma$ has at worst plane curve singularites at $P, \mathcal{I}_{\Gamma, \mathrm{C}^{4}}$ is generated by a regular sequence $\left\{g_{1}, g_{2}, g_{3}\right\}$. Hence $B_{\Gamma} \mathrm{C}^{4} \longrightarrow \mathrm{C}^{4}$ is the blow up of a regular sequence and thus all its fibers over $\Gamma$ are isomorphic to $\mathbb{P}^{2}$. Hence $E_{2} \cong \mathbb{P}^{2}$.

LEMMA 2.4. Let $P \in \Gamma \subset S$. Suppose that $P \in \Gamma$ is at worst a plane curve singularity, $\Gamma-P$ is smooth, and $S$ is a normal and canonical surface. Let $f: S^{\prime}=B_{\Gamma} S \longrightarrow S$ be the blow up of $S$ along $\Gamma$. Then $S^{\prime}$ is normal.

Proof. Clearly, $S^{\prime}-f^{-1}(P) \cong S-P$ since it is the blow up of a Cartier divisor. So the result is local over $P$. Hence, we can assume that $S \subset X=\mathrm{C}^{3}$. Let $f: Y=B_{\Gamma} X \longrightarrow X$ be the blow up of $X$ along $\Gamma$, and $E$ the $f$-exceptional divisor. Since $P \in \Gamma$ is a lci singularity, $Y$ is just the blow up of a regular sequence, say $\left\{g_{1}, g_{2}\right\}$ in $X$. Hence, $E \cong \mathbb{P}_{\Gamma}\left(I_{\Gamma, X} / I_{\Gamma, X}^{2}\right)$. In particular, $\operatorname{dim} f^{-1}(x) \leqslant 1 \forall x \in X$. Hence, since $Y$ is the blow up of a smooth curve away from $P, Y$ is normal. Moreover, $f^{*} S=S^{\prime}+E$, and $K_{Y}=f^{*} K_{X}+E$. Hence

$$
K_{Y}+S^{\prime}=f^{*}\left(K_{X}+S\right) .
$$

By [Ko97, Theorem 7.3], the pair $(X, S)$ is also canonical. Hence, $\left(Y, S^{\prime}\right)$ is also canonical and, hence, plt. By [Ko-Mo98, Theorem 5.51] and [Ko97], $S^{\prime}$ is normal and canonical.

LEMMA 2.5. Let $P \in \Gamma \subset X$. Assume that $\Gamma$ is smooth and that $P \in X$ is a 3dimensional normal hypersurface singularity. Let $f: Y=B_{\Gamma} X \longrightarrow X$ be the blow up of $X$ along $\Gamma$. Let $P \in S \subset X$ be a general hypersurface section through $P$. Then

$$
f^{*} S=S^{\prime}+\left(m_{P} X-1\right) E_{2},
$$

where $m_{P} S$ is the multiplicity of $S$ at $P$ and $f^{-1}(\Gamma)=E_{1}+\mathrm{d} E_{2}$ as in Lemma 2.3.

Proof. Suppose that $f^{*} S=S^{\prime}+a E_{2} \cdot f^{-1}(\Gamma)=E_{1}+\mathrm{d} E_{2}$ is Cartier and $\mathcal{O}_{Y}\left(E_{1}+\right.$ $\left.\mathrm{d} E_{2}\right)=\mathcal{O}_{Y}(-1)$. Then,

$$
f^{*} S \cdot\left(E_{1}+\mathrm{d} E_{2}\right)^{2}=S^{\prime} \cdot\left(E_{1}+\mathrm{d} E_{2}\right)^{2}+a E_{2} \cdot\left(E_{1}+\mathrm{d} E_{2}\right)^{2}
$$


Since $E_{2} \cong \mathbb{P}^{2}$, it follows that $E_{2} \cdot\left(E_{1}+\mathrm{d} E_{2}\right)^{2}=1$. Moreover, $S^{\prime}=B_{P} S$ the blow up of $S$ at $P$. Let $F$ be the exceptional divisor. Then $F^{2}=-m_{P} S=-m_{P} X$, and $F^{2}=S^{\prime} \cdot\left(E_{1}+\mathrm{d} E_{2}\right)^{2}$. Moreover, $\left(E_{1}+\mathrm{d} E_{2}\right)^{2}=E_{1} \cdot\left(E_{1}+\mathrm{d} E_{2}\right)+\mathrm{d} E_{2} \cdot\left(E_{1}+\mathrm{d} E_{2}\right)=$ $\Delta+\mathrm{d} L$, where $\Delta$ is a section of $E_{1} \longrightarrow \Gamma$ and $L$ a line in $E_{2}=\mathbb{P}^{2}$. Hence $f^{*} S \cdot\left(E_{1}+\mathrm{d} E_{2}\right)^{2}=S \cdot \Gamma=1$, and therefore $a=m_{P} S-1=m_{P} X-1$.

PROPOSITION 2.6. Let $P \in \Gamma \subset X$. Assume that $P \in X$ is an index 1 terminal singularity and $X$ normal. let $f: Y=B_{\Gamma} X \longrightarrow X$. Then:

(1) If $\Gamma$ is smooth, then $Y$ is normal of index 1.

(2) If there is a DuVal section $P \in \Gamma \subset S \subset X$ and $\Gamma$ has at worst plane curve singularities, then $Y$ is normal and canonical of index 1 .

(3) $K_{Y}=f^{*} K_{X}+E_{1}+d E_{2}$, and $f^{-1}(\Gamma)=E_{1}+\mathrm{d} E_{2}$ is Cartier but $E_{1}, E_{2}$ are not Q-Cartier.

Proof. First we will show that $Y$ is $\mathrm{CM}$ and that $\omega_{Y}$ is invertible. The result is local around $P$. Since $P \in X$ is index 1 terminal, it is cDV. So we can assume that $X \subset \mathrm{C}^{4}=U$. Let $W=B_{\Gamma} \mathrm{C}^{4}$. Since $\Gamma$ has at worst plane curve singularities, $W$ is the blow up of a regular sequence and therefore $W$ is lci and hence $\mathrm{CM}$ and $\omega_{W}$ is invertible. Moreover, $E=f^{-1}(\Gamma)=E \cong \mathbb{P}_{\Gamma}\left(I_{\Gamma, U} / I_{\Gamma, U}^{2}\right)$ is Cartier and irreducible. Suppose that $f^{*} X=Y+a E, a \in \mathbb{N}$. Hence $Y \subset W$ is Cartier and hence is $C M$ and $\omega_{Y}$ is invertible.

Now assume that $\Gamma$ is smooth. Since $P \in X$ is $\mathrm{cDV}, m_{P} X=2$. Let $P \in S \subset X$ be a general hypersurface section. Then $P \in S$ is DuVal. From Lemma 2.5 it follows that $f^{*} S=S^{\prime}+E_{2}$. Since $E_{2} \cong \mathbb{P}^{2}$ and $S^{\prime}+E_{2}$ is Cartier, it follows that $Y$ is smooth at the generic point of $E_{2}$ and hence regular in codimension 1 and therefore normal. This shows 1. Now suppose that $f^{-1}(\Gamma)=E_{1}+d E_{2}$. This is of course Cartier. Since $Y \subset W$ is Cartier, we can use adjunction to calculate $K_{Y}$. Since $W-f^{-1}(P)$ is the blow up of a smooth curve, it follows that $K_{W}=f^{*} K_{U}+2 E$. Moreover, $f^{*} X=Y+E$. Hence

$$
\begin{aligned}
K_{Y}=\left.\left(K_{W}+Y\right)\right|_{Y} & =f^{*}\left(K_{U}+X\right)+\left.E\right|_{Y}=f^{*} K_{X}+\left.E\right|_{Y} \\
& =f^{*} K_{X}+E_{1}+\mathrm{d} E_{2} .
\end{aligned}
$$

If $E_{i}$ were Q-Cartier, then for a fiber $\delta$ of $f$ disjoint from $E_{2}$ and a line $l \subset E_{2}, \delta=a l$, $a \geqslant 0$. Hence $0 \leqslant l \cdot E_{1}=a \delta \cdot E_{1}=a \delta \cdot\left(E_{1}+\mathrm{d} E_{2}\right)=-a<0$, which is not possible. Of course one could argue that in this case the exceptional would have to be irreducible which is not the case.

Now suppose that a DuVal section $\Gamma \subset S \subset X$ exists. Then $S=X \cap H$ for some general plane in $U$. Therefore, $f^{*} H=H^{\prime}+E$ and hence it follows that $f^{*} S=S^{\prime}+E_{1}+\mathrm{d} E_{2}$. In particular, $S^{\prime}$ is Cartier. From Lemma 2.4 it follows that $S^{\prime}$ is normal and canonical and therefore since $S^{\prime}$ is Cartier, $Y$ is smooth at some points of $E_{2}$ and hence regular in codimension 1 and therefore normal. Since $X$ is 
terminal and $S$ is canonical it follows that $(X, S)$ is canonical. Adjunction for $S^{\prime}$ in $Y$ gives that $K_{Y}+S^{\prime}=f^{*}\left(K_{X}+S\right)$. Hence, the pair $\left(Y, S^{\prime}\right)$ is also canonical. Since $Y$ has index 1 and $S^{\prime}$ is DuVal, it follows that $Y$ is also canonical.

If there is no DuVal section $S$ containing $\Gamma$, then $Y$ may fail to be canonical. In fact as shown by Example 1.5, it may not even be log canonical.

\section{Proof of Theorem 1.6}

Let $f: Y=B_{\Gamma} X \longrightarrow X$. Then by Proposition 2.6, $Y$ is canonical and normal. Therefore by [Kaw88], there is $g: Z \longrightarrow Y$ such that $g$ is an isomorphism in codimension 1 and $-E_{1}^{Z}=g_{*}^{-1} E_{1}$ is Q-Cartier and $g$-ample. More precisely, $R\left(E_{1}, Y\right)=\bigoplus_{i} \mathcal{O}_{Y}\left(-i E_{1}\right)$ is finitely generated and $Z=\operatorname{Proj} R\left(E_{1}, Y\right)$. Hence, $K_{Z}=g^{*} K_{Y}$ and $Z$ is canonical. In fact since $X$ is Q-factorial, $Z$ is also Qfactorial. We want to contract $E_{2}^{Z}=g_{*}^{-1} E_{2}$ over $X$ and obtain the required contraction.

Let $S$ be the general hyperplane section of $X$ containing $\Gamma$. Then as we have seen in the proof of Proposition 2.6, $K_{Y}+S^{Y}=f^{*}\left(K_{X}+S\right)$ and therefore $K_{Z}+S^{Z}=$ $h^{*}\left(K_{X}+S\right)$, where $h=g \circ f$. Now run a $\left(Z, S^{Z}+\epsilon E_{2}^{Z}\right)$ MMP over $X$. We get a diagram

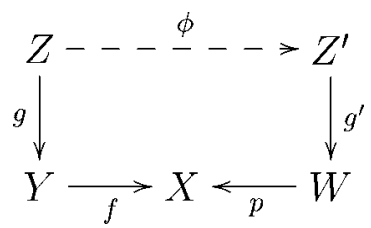

where $\phi$ is a composition of flips and flops, and $g^{\prime}$ is divisorial. A this point I must say that in all cases that I have worked explicitely, there are no flips or flops. Since the first step of the MMP is the contraction of a $K_{Z}$-negative extremal ray, this would follow from [Benv85, Theorem 0]. However, Takagi pointed out an example to me that shows that this theorem is not correct.

The only thing left is to show that $W$ is canonical. Since $\phi$ is an isomorphism in codimension 1 , it follows that $K_{Z^{\prime}}+S^{Z^{\prime}}=\left(h^{\prime}\right)^{*}\left(K_{X}+S\right)$ where $h^{\prime}=p \circ g^{\prime}$. Therefore, since $(X, S)$ is canonical, $Z^{\prime}$ is also canonical. Moreover, if $C$ is a $g^{\prime}$ exceptional curve, then $S^{Z^{\prime}} \cdot C \geqslant 0$ and therefore $K_{Z^{\prime}} \cdot C=-S^{Z^{\prime}} \cdot C \leqslant 0$. Now by [Ko-Mo98, Proposition 3.38], it follows that $W$ is also canonical

Therefore, in order to understand when $W$ is terminal, it is important to describe the Q-factorialization of $E_{1}, Z$. If there are no flops or flips, i.e., $Z=Z^{\prime}$ (in particular, this is the case when none of the $g$-exceptional curves is contained in $E_{2}^{Z}$ ), $W$ is terminal iff $Z$ has isolated terminal singularities away from $E_{2}^{Z}$ [Ko-Mo98, Proposition 3.38]). 
In general, describing explicitely $Z$ is difficult. However, in the cases that the general section $S$ of $X$ through $\Gamma$ is $D_{2 n}$ or $A_{i}$ with $i \leqslant 3$, it is possible to get such a description and therefore treat these cases completely.

\section{Normal Forms for $\Gamma \subset S \subset X$}

In this section we will obtain normal forms for the equation of $\Gamma \subset S \subset X$. For the rest of this paper $\Gamma$ will be smooth. Jaffe [Jaf92] obtained a classification of pairs $(S, \Gamma)$ when $S$ is DuVal and $\Gamma \subset S$ a smooth curve. Let $U \longrightarrow S$ be the minimal resolution of $S$ and let $E_{i}$ be the exceptional curves. The next lemma gives normal forms for the equations of $\Gamma$ in $S$ based on the position of $\Gamma$ in the fundamental cycle. It essentially follows from [Jaf92].

LEMMA 4.1. With assumptions as before.

(1) Suppose that $0 \in S$ is $A_{n}$, and that the fundamental cycle is

$$
\begin{aligned}
& \stackrel{E_{1}}{\circ}-\stackrel{E_{2}}{\circ}-\cdots-\stackrel{E_{n-1}}{\circ}-\stackrel{E_{n}}{\circ} . \\
& \text { If } \Gamma \text { intersects the } E_{k} \text { then } S \text { is given by } x y-z^{n+1}=0 \text { and } \Gamma \text { by } \\
& I=\left(x-z^{k}, y-z^{n+1-k}\right), 1 \leqslant k \leqslant(k+1) / 2 .
\end{aligned}
$$

(2) Suppose that $0 \in S$ is $D_{n}$ and that the fundamental cycle is

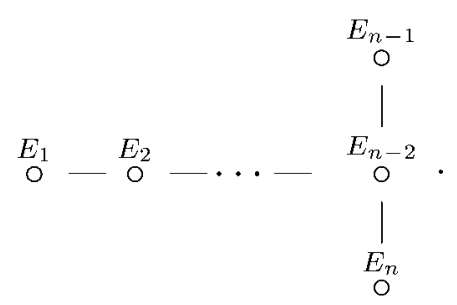

$\Gamma$ can only intersect $E_{1}, E_{n-1}$ or $E_{n}$. If it intersects $E_{1}$ then it is given by $I=(x, z)$ and $S$ by $x^{2}+y^{2} z+z^{n-1}=0$. If it intersects $E_{n-1}$ or $E_{n}$, then by an obvious change of variables:

(a) In the $D_{2 n}$ case, $S$ is given by $x^{2}+y^{2} z+2 y z^{n}=0$ and $\Gamma$ by $I=(x, y)$.

(b) In the $D_{2 n+1}$ case, $S$ is given by $x^{2}+y^{2} z+2 x z^{n}=0$ and $\Gamma$ by $I=(x, y)$.

DEFINITION 4.2. Let $\Gamma$ be a smooth curve on a surface $S$. Suppose that $S$ has exactly one singular point on $\Gamma$ which is of type $D_{n}$. Let $f: U \longrightarrow S$ be its minimal resolution and $\Gamma^{\prime}=f_{*}^{-1} \Gamma$. Then:

(1) $\Gamma \subset S$ will be called of type $F D_{l}$ if $\Gamma^{\prime}$ intersects $E_{1}$ in the minimal resolution of $S$.

(2) $\Gamma \subset S$ will be called of type $F D_{r}$ if $\Gamma^{\prime}$ intersects $E_{n}$ or $E_{n-1}$.

Next we will derive the simplest possible normal forms for $0 \in \Gamma \subset S \subset X$. To do this it is necessary to obtain some properties of $S$. 
First we will show that if $0 \in X$ is $c A_{n}$ then $0 \in S$ is $A_{m}$ for the general section $S$ of $X$ containing $\Gamma$.

LEMMA 4.3. Let $0 \in \Gamma \subset X, \Gamma$ a smooth curve and $0 \in X$ a c $A_{n}$ 3-fold singularity. Then the general hyperplane section $S$ of $X$ containing $\Gamma$ is $A_{m}$.

Proof. Since $\Gamma$ is smooth, we can assume that it is given by $x=y=z=0$, and $X$ by

$$
q(x, y, z)+t\left[\sum_{k} \phi_{k}(x, y, z) t^{k}\right]=0
$$

and the section given by $t=0$ is $A_{n}$. Therefore

$$
q(x, y, z)=q_{2}(x, y, z)+\sum_{i \geqslant 3} q_{i}(x, y, z)
$$

with $q_{2}(x, y, z)$ a quadratic that is not a square.

A general hyperplane through $\Gamma$ is $x=b y+c z$. Then $S$ is given by

$$
q_{2}(b y+c z, y, z)+\sum_{i \geqslant 3} q_{i}(b y+c z, y, z)+t\left[\sum_{I} \phi_{i}(b y+c z, y, z) t^{i}\right]=0 .
$$

It's quadratic term is

$$
\psi_{2}=q_{2}(b y+c z, y, z)+t l(b y+c z, y, z)+a t^{2}
$$

where $l(b y+c z, y, z)$ is linear. $0 \in S$ is $A_{n}$ iff $\psi_{2}$ is not a square. If it is a square, then putting $t=0$ it follows that $q_{2}(b y+c z, y, z)$ is also a square.

Claim. If $q_{2}(b y+c z, y, z)$ is a square for all $b, c$ then $q_{2}(x, y, z)$ is also a square. To see this suppose that

$$
q_{2}(x, y, z)=a_{1} x^{2}+a_{2} y^{2}+a_{3} z^{2}+a_{4} x y+a_{5} x z+a_{6} y z .
$$

Then

$$
\begin{aligned}
& q_{2}(b y+c z, y, z) \\
& =\left(a_{1} b^{2}+a_{2}+a_{4} b\right) y^{2}+\left(a_{1} c^{2}+a_{3}+a_{5} c\right) z^{2}+\left(2 b c a_{1}+a_{4} c+a_{5} b+a_{6}\right) y z .
\end{aligned}
$$

This is a square iff

$$
4\left(a_{1} c^{2}+a_{3}+a_{5} c\right)\left(a_{1} b^{2}+a_{2}+a_{4} b\right)=\left(2 b c a_{1}+a_{4} c+a_{5} b+a_{6}\right)^{2} \quad \forall b, c .
$$

Hence, $a_{1}=a_{4}=a_{5}=0$ and $4 a_{2} a_{3}=a_{6}^{2}$. But then $q_{2}(x, y, z)=a_{2} y^{2}+a_{3} z^{2}+a_{6} y z$ is a square.

Now let $f: Y=B_{\Gamma} X \rightarrow X$ as before. Let $f^{-1}(\Gamma)=E_{1}+\mathrm{d} E_{2}$. We want to relate $d$ with some quantity on the general hyperplane section $S$ of $X$, through $\Gamma$. 
LEMMA 4.4. Let $f: Y=B_{\Gamma} X \longrightarrow X$. Assume that $Y$ is canonical. Suppose that $f^{-1}(\Gamma)=E_{1}+\mathrm{d} E_{2}$. Then there is a Du Val section $S$ of $X$ containing $\Gamma$ such that if $g: S^{\prime}=f_{*}^{-1} S \longrightarrow S$, then $g^{-1}(\Gamma)=\Gamma^{\prime}+\mathrm{d} E$ and $E \cong \mathbb{P}^{1}$ is the g-exceptional curve. In fact this is true for the general section $S$ of $X$ through $\Gamma$.

This way we see that somehow $d$ controls the type of the general hyperplane section through $\Gamma$. This result will be useful later when we try to get normal forms for the equations of $0 \in \Gamma \subset S \subset X$.

Proof. Let $Q \in L=E_{1} \cap E_{2}$ be a cDV point and let $S^{\prime} \subset Y$ be general through $Q$. Then $Q \in S^{\prime}$ is DuVal. Since $S^{\prime}$ is general, $S^{\prime} \cap\left(E_{1}+\mathrm{d} E_{2}\right)=\Gamma^{\prime}+\mathrm{d} E$, and $\Gamma^{\prime}$ maps to $\Gamma$. Moreover, for a general $\delta \subset E_{1}, \delta \cdot S^{\prime}=1$ and, hence, $\delta \cdot\left(S^{\prime}+E_{1}+\mathrm{d} E_{2}\right)=0$. Therefore, there is a Cartier divisor $S, 0 \in \Gamma \subset S \subset X$ such that $f^{*} S=$ $S^{\prime}+E_{1}+\mathrm{d} E_{2}$. By adjunction

$$
K_{S^{\prime}}=\left.\left(K_{Y}+S^{\prime}\right)\right|_{S^{\prime}}=\left.f^{*}\left(K_{X}+S\right)\right|_{S^{\prime}}=f^{*} K_{S} .
$$

Therefore $S$ is canonical with the required property.

The next proposition relates $d$ with the type of singularities of $S$.

PROPOSITION 4.5. Let $P \in \Gamma \subset S . \Gamma$ a smooth curve and $P \in S$ Du Val. Let $g: S^{\prime} \longrightarrow S$ be the blow up of $S$ along $\Gamma$. Let $E$ be the g-exceptional curve which is necessarily irreducible. Suppose that $g^{-1}(\Gamma)=\Gamma^{\prime}+\mathrm{d} E$. Let $f: U \longrightarrow S$ be the minimal resolution of $S, E_{i}$ the f-exceptional curves and $\Gamma^{\prime \prime}$ the birational transform of $\Gamma$ in $U$. Then

(1) Suppose that $P \in S$ is $A_{n}$ and that $\Gamma^{\prime \prime}$ intersects $E_{k}, 1 \leqslant k \leqslant(n+1) / 2$. Then

(a) $\left[f^{-1}(\Gamma)\right]=\Gamma^{\prime \prime}+\sum_{i=1}^{k-1} i E_{i}+k \sum_{i=k}^{n-k+1} E_{i}+\sum_{i=1}^{k-1}(k-i) E_{n-k+i+1}$.

(b) $\mathrm{E}=\mathrm{E}_{\mathrm{n}-\mathrm{k}+1}$, and $d=k$.

(2) Suppose that $P \in S$ is $D_{n}$. Then $\Gamma^{\prime \prime}$ intersects one of the edges of the dual graph of S. Then

(a) With notation as in Lemma 4.2.2, if $\Gamma^{\prime \prime}$ intersects $E_{1}$, then $E=E_{1}$ and $d=2$. Moreover $S^{\prime}$ is smooth along $\Gamma^{\prime}$ and

$$
\left[f^{-1}(\Gamma)\right]=\Gamma^{\prime \prime}+2 \sum_{i=1}^{n-2} E_{i}+E_{n-1}+E_{n}
$$

(b) If $\Gamma^{\prime \prime}$ intersects $E_{n-1}$ then

(i) if $n$ is even, then $E=E_{n-1}, d=n / 2$, is smooth along $\Gamma^{\prime}$ and

$$
\left[f^{-1}(\Gamma)\right]=\Gamma^{\prime \prime}+E_{1}+2 \sum_{i=2}^{n-2} E_{i}+\frac{n}{2} E_{n-1}+\frac{n-2}{2} E_{n}
$$


(ii) if $n$ is odd, then $E=E_{n}, d=(n-1) / 2$, and

$$
\left[f^{-1}(\Gamma)\right]=\Gamma^{\prime \prime}+E_{1}+2 \sum_{i=2}^{n-2} E_{i}+\frac{n-1}{2} E_{n-1}+\frac{n-1}{2} E_{n} .
$$

COROLLARY 4.6. Let $P \in \Gamma \subset S \subset X$, $\Gamma$ smooth and $P \in X$ a cDV point. Let $f: Y=B_{\Gamma} X \longrightarrow X, f^{-1}(\Gamma)=E_{1}+\mathrm{d} E_{2}$ and $L=E_{1} \cap E_{2}$. If $P \in S$ is a $D_{2 n}$ singularity, then $Y$ has finitely many singular points on $L$ and therefore $a \mathrm{Q}$-factorialization of $E_{1}$ is obtained by blowing up $E_{1}$.

Proof. From the previous proposition it follows that $S^{\prime}=f_{*}^{-1} S$ is smooth at one point of $L$. Therefore since $S^{\prime}$ is Cartier, $Y$ is also smooth at this point and hence has finitely many singularities along $L$.

Proof Proposition 4.5. Let $Z=\left[f^{-1}(\Gamma)\right]$. This is an integral cycle. From the properties of the blow up it follows that $g^{-1}(\Gamma)$ is Cartier and, hence, $Z \cdot E=-1$ and $Z \cdot E_{i}=0$ for all $i$ such that $E_{i} \neq E$. Moreover, $d$ is just the coefficient of $E$ in $Z$.

We will only do the case when $0 \in S$ is $D_{n}$, with $n$ odd, and $\Gamma^{\prime \prime}$ intersects $E_{n-1}$. The rest is similar. In fact the $A_{n}$ is simpler.

Observe that since $S$ has embedding dimension 4 and $S^{\prime}$ is the blow up of a smooth curve, it follows that $g^{-1}(0)=E=\mathbb{P}^{1}$. Therefore $E$ appears with coefficient 1 in the fundamental cycle and, hence, it must be one of the edges. So let $Z=\Gamma^{\prime \prime}+\sum_{i} a_{i} E_{i}$, $a_{i} \in \mathbb{N}$. There are three cases to be considered. Only one will give an integral cycle and this will be the answer. We will only work the case that $E=E_{n}$. The others are similar.

The relations $Z \cdot E_{n}=-1$ and $Z \cdot E_{i}=0, \forall i \neq n$ give the system of equations

$$
\begin{aligned}
& a_{k-1}-2 a_{k}+a_{k+1}=0, \quad 1 \leqslant k \leqslant n-2, \\
& a_{n-3}-2 a_{n-2}+a_{n-1}+a_{n}=0, \\
& a_{n-2}-2 a_{n-j+1}+1=0, \quad j=1,2 .
\end{aligned}
$$

It is easy to see that the solution of this system is $a_{i}=i$ for $2 \leqslant i \leqslant n-2$, and $a_{n-1}=a_{n}=(n-1) / 2$. This solution gives an integral cycle. Similarly we see that the cases $E=E_{n-1}$ and $E=E_{1}$ do not give integer cycles and, hence, are not possible.

Therefore,

$$
\left[f^{-1}(\Gamma)\right]=\Gamma^{\prime \prime}+E_{1}+2 \sum_{i=2}^{n-2} E_{i}+\frac{n-1}{2} E_{n-1}+\frac{n-1}{2} E_{n},
$$

$E=E_{n}$ and $d=(n-1) / 2$.

We are now in position to get normal forms for the equations $0 \in \Gamma \subset S \subset X$ in the case that $0 \in S$ is $D_{n}$ and $S$ is general through $\Gamma$. We will not treat the general 
$A_{n}$ case here and so I will not attempt to write normal forms in this case. However, normal forms for the case when $0 \in S$ is $A_{3}$ will be given in the proof of Theorem 5.1.

PROPOSITION 4.7. Suppose $0 \in \Gamma \subset S \subset X$, $S$ general. Suppose that $0 \in S$ is $D_{n}$. Then under suitable choice of coordinates, $0 \in \Gamma \subset S \subset X$ is given by

(1) If $\Gamma \subset S$ is of type $F D_{l}$, then

$$
X: x^{2}+y^{2} z+z^{n-1}+t \phi_{\geqslant 2}(y, z, t)=0, I_{\Gamma}=(x, z, t) .
$$

Moreover, no $y^{k}$ appears in $\phi_{\geqslant 2}(y, z, t)$ for any $k$. For $n=4$ this is the only possibility.

$$
\text { If } n \geqslant 5 \text { then } \phi_{2}=0 \text {. That is } X \text { is given by }
$$

$$
x^{2}+y^{2} z+z^{n-1}+t \phi_{\geqslant 3}(y, z, t)=0
$$

and again no $y^{k}$ appears in $\phi_{\geqslant 3}(y, z, t)$.

(2) Suppose that $\Gamma \subset S$ is of type $F D_{r}$. Then

(a) If $n$ is even then $0 \in \Gamma \subset S \subset X$ is given by

$$
x^{2}+y^{2} z+2 y z^{n / 2}+t \phi_{\geqslant 2}(y, z, t)=0, I_{\Gamma}=(x, y, t) .
$$

Moreover, There is no $z^{k}$ in $\phi_{\geqslant 2}(y, z, t)$ for any $k$.

(b) If $n$ is odd then $0 \in \Gamma \subset S \subset X$ is given by

(i) $x^{2}+y^{2} z+2 x z^{\frac{n-1}{2}}+t\left[a x z^{k}+\phi_{\geqslant 2}(y, z, t)\right]=0, k \geqslant 1 I_{\Gamma}=(x, y, t)$.

No $y z$ or $z^{v}$ appear in $\phi_{\geqslant 2}(y, z, t)$ for any $v . a \in \mathbb{Z}$.

(ii) Alternatively, the equation can take the form

$$
\begin{aligned}
& x^{2}+y^{2} z+2 x z^{\frac{n-1}{2}}+t\left[x z \psi(z, t)+a x t^{\frac{n-3}{2}}+b x t^{k}+\phi_{\geqslant 2}(y, z, t)\right]=0, \\
& k \geqslant 1, \quad I_{\Gamma}=(x, y, t) \text { and in this case, } y^{2}, y z \text {, or } z^{v} \text { do not appear in } \\
& \phi_{\geqslant 2}(y, z, t), \text { for any } v .
\end{aligned}
$$

Sometimes it is better to have $2 . b$ and sometimes 2.a. The existence of $y^{2}$ may complicate calculations.

Proof. We will apply the following methods:

(1) The Weierstrass preparation theorem.

(2) The elimination of the $y^{n-1}$-term from the polynomial $a_{n} y^{n}+a_{n-1} y^{n-1}+\cdots$ by a coordinate change $y \longrightarrow y-a_{n-1} / n a_{n}$ when $a_{n}$ is a unit.

(3) Let $M_{1}, M_{2}, M_{3}, M_{4}$ be multiplicatively independent monomials in the variables $x, y, z, t$. Then any power series of the form $M_{1} \cdot$ (unit) $+M_{2} \cdot$ (unit) $+M_{3} \cdot$ (unit) $+M_{4}$. (unit) is equivalent to $M_{1}+M_{2}+M_{3}+M_{4}$ by a suitable coordinate change $x \mapsto x \cdot$ (unit), $y \mapsto y \cdot$ (unit), $z \mapsto z \cdot$ (unit), $t \mapsto t \cdot$ (unit). 
I will only present here the proof of (1). The rest can be proved by the same method.

So, suppose that $\Gamma^{\prime \prime}$ intersects $E_{1}$ in the dual graph. Then by Lemma 4.1, $0 \in \Gamma \subset X$ is given by

$$
x^{2}+y^{2} z+z^{n-1}+t \phi_{\geqslant 1}(x, y, z, t)=0
$$

and $\Gamma$ is given by $x=z=t=0$. Apply the Weierstrass preparation theorem on $x^{2}$ to eliminate $x$ from $\phi$. Moreover, by Lemma 4.4, if $\phi$ has linear terms, then $0 \in X$ is $c A_{n}$ and therefore the general section $\Gamma \subset S \subset X$ is $A_{m}$ which is not possible by our assumptions. Therefore $\Gamma \subset S \subset X$ is given by

$$
x^{2}+y^{2} z+z^{n-1}+t \phi_{\geqslant 2}(y, z, t)=0,
$$

and $\Gamma=(x, z, t)$.

Now suppose that

$$
\phi_{\geqslant 2}(y, z, t)=f_{\geqslant 2}(y)+\Phi_{\geqslant 2}(y, z, t),
$$

with no $y^{k}$ in $\Phi_{\geqslant 2}(y, z, t)$. Then write $f(y)=y^{k}$. (unit), $k \geqslant 2$. Then the equation of $X$ is

$$
x^{2}+y^{2}\left(z+t y^{k-2} \cdot(\text { unit })\right)+z^{n-1}+t \Phi_{\geqslant 2}(y, z, t)=0 .
$$

The change of variables $z \mapsto z-t y^{k-2}$. (unit) will give the normal form claimed by the first part of 1 .

Now suppose that $n \geqslant 5$. If $\phi_{2}(y, z, t)=0$ then there is nothing to prove. Suppose it is not zero. Then we already know that in the $D F_{l}$ case it is possible to write the equations of $\Gamma \subset S \subset X$ as

$$
G=x^{2}+y^{2} z+z^{n-1}+t \phi_{\geqslant 2}(y, z, t)=0,
$$

and no power of $y, y^{k}$, appears in $\phi_{\geqslant 2}(y, z, t)$. Let

$$
\phi_{\geqslant 2}(y, z, t)=\phi_{2}(y, z, t)+\phi_{\geqslant 3}(y, z, t) .
$$

Let $\phi_{2}(y, z, t)=a_{1} z^{2}+a_{2} t^{2}+a_{3} y z+a_{4} y t+a_{5} z t$. Let $S$ be the section given by $t=\lambda z$. This is given by

$$
F(x, y, z)=x^{2}+y^{2} z+z^{n-1}+\lambda z \phi_{2}(y, z, \lambda z)+\lambda z \phi_{\geqslant 3}(y, z, \lambda z)=0,
$$

and $\phi_{2}(y, z, \lambda z)=\left(a_{1}+a_{2} \lambda^{2}+a_{5} \lambda\right) z^{2}+\left(a_{3}+a_{4} \lambda\right) y z$. If $\phi_{2} \neq 0$, then for general $\lambda$ at least one of the coeffiecients of $z^{2}$ or $y z$ in not zero. Suppose that both coefficients are nonzero. i.e., $a=a_{1}+a_{2} \lambda^{2}+a_{5} \lambda \neq 0$, and $b=a_{3}+a_{4} \lambda \neq 0$. Hence $\phi_{2}(y, z, \lambda z)=$ $a z^{2}+b y z, a b \neq 0$ and

$$
F=x^{2}+y^{2} z+z^{n-1}+\lambda z\left(a z^{2}+b y z\right)+\lambda z \phi_{\geqslant 3}(y, z, \lambda z) .
$$

Now write

$$
\phi_{\geqslant 3}(y, z, \lambda z)=z f_{\geqslant 2}(y)+z^{2} f_{\geqslant 1}(y, z, \lambda z), \quad f_{\geqslant 2}(y)=y^{k} f(y), k \geqslant 2
$$


Then

$$
\begin{aligned}
F & =x^{2}+y^{2} z+y z^{2}\left[\lambda b+\lambda y^{k-1} f(y)\right]+z^{3}\left[\lambda a+z^{n-4}+\lambda f_{\geqslant 1}(y, z, \lambda z)\right] \\
& =x^{2}+y^{2} z+y z^{2} \cdot u_{1}+z^{3} \cdot u_{2}
\end{aligned}
$$

where $u_{1}=\lambda b+\lambda y^{k-1} f(y), u_{2}=\lambda a+z^{n-4}+\lambda f_{\geqslant 1}(y, z, \lambda z)$, are units. Hence $F=x^{2}+$ $z\left[y^{2}+y z \cdot u_{1}+z^{2} \cdot u_{2}\right]$. Eliminate the $y z$-term by the change of variables $y \mapsto y-z u_{1} / 2$. Hence the equation becomes

$$
F=x^{2}+z\left[y^{2}-\frac{z^{2} u_{1}^{2}}{4}+z^{2} u_{2}\right]=x^{2}+y^{2} z+z^{3}\left(u_{2}-\frac{u_{1}^{2}}{4}\right) .
$$

We will now consider cases with respect to the nature of $\delta=u_{2}-\left(u_{1}^{2} / 4\right)$.

Suppose that $\delta$ is a unit. Then $F \sim x^{2}+y^{2} z+z^{3}$ and therefore $S$ is $D_{4}$.

Suppose now that $\delta$ is not a unit. First check when this happens. By looking at how $u_{1}, u_{2}$ are defined, we see that

$$
\delta=\left(\lambda a-\frac{\lambda^{2} b^{2}}{4}\right)+\{\text { higher }\}
$$

Therefore $\delta$ is not a unit iff $a-\left(\lambda b^{2} / 4\right)=0, \quad \forall \lambda$. But this implies that $a_{1}+\lambda^{2} a_{2}+$ $\lambda a_{5}=\frac{1}{4} \lambda\left(a_{3}+a_{4} \lambda\right)^{2}$, for all $\lambda$, and hence $a_{1}=a_{2}=a_{4}=4 a_{5}-a_{3}^{2}=0$. Therefore $\phi_{2}(y, z, t)=\frac{1}{4} a^{2} z t+a y z$ Then

$$
\begin{aligned}
G & =x^{2}+y^{2} z+z^{n-1}+t\left(\frac{1}{4} a^{2} z t+a y z\right)+t \phi_{\geqslant 3}(y, z, t) \\
& =x^{2}+z\left(y^{2}+\frac{1}{4} a^{2} t^{2}+a y t\right)+z^{n-1}+t \phi_{\geqslant 3}(y, z, t) \\
& =x^{2}+z\left(y+\frac{1}{2} a t\right)^{2}+z^{n-1}+t \phi_{\geqslant 3}(y, z, t) .
\end{aligned}
$$

Make the change of variables $y \mapsto y-\frac{1}{2}$ at to bring the equation in the form

$$
x^{2}+y^{2} z+z^{n-1}+t \phi_{\geqslant 3}(y, z, t)=0,
$$

which is what we want.

Similar arguments show that if $a=0$ or $b=0$ for all $\lambda$, then a $D_{4}$ section exists. This concludes the proof of the proposition.

\section{The $A_{1}, A_{2}$, and $A_{3}$ Cases}

In this section we will study the existence of terminal contractions in the case when $P \in S$ is $A_{1}, A_{2}$ or $A_{3}$.

THEOREM 5.1. Let $P \in \Gamma \subset S \subset X$. Suppose that $P \in S$ is $A_{n}$. Then

(1) Suppose that $\Gamma$ intersects one edge of the dual graph of the singularities of S. (In particular, this is always the case when $n=1,2)$. Then there is always a terminal 
contraction $E \subset W \longrightarrow \Gamma \subset X$ contracting a surface $E$ to $\Gamma$. If $n=1$ then $W$ has exactly one non Gorenstein point which is a $\frac{1}{2}(1,1,1)$ singularity.

If $n>1$, then

$2 \leqslant \operatorname{index}(W) \leqslant n+1$.

In the case that the central curve is irreducible we can say more. In particular, there are two cases

(a) Whas exactly one non Gorenstein point which is a cyclic quotient singularity of index $n+1$

(b) $W$ has exactly 2 non Gorenstein points and it is of IA $+I A$ type (in the sense of [Ko-Mo92]). Let $S^{W}$ be the birational transform of $S$ in $W$. It has one $A_{r}$ and one $A_{s}$ singular point. Moreover, $r, s$ are the indices of the two singular points of $W, k r+l s-1=n$, and $k, l$ their axial multiplicities.

(2) Suppose that $n=3$. Then

(a) If $\Gamma$ intersects one edge of the dual graph of the singularities of $S$, then there is a terminal contraction as follows from the previous part.

(b) If $\Gamma^{\prime \prime}$ intersects $E_{2}$, i.e. the middle of the dual graph, then write the equation of $\Gamma \subset S \subset X$ as

$x^{2}+y^{2}+f_{\leqslant 3}(y, z, t)+f_{\geqslant 4}(y, z, t)=0$,

no $y^{2}$ appears in $f_{\leqslant 3}(y, z, t)$ and $I_{\Gamma}=(x, y, t)$. (This is always possible.) Then a terminal contraction exists iff $f_{\leqslant 3}(y, z, t)$ is irreducible, and it has exactly one non-Gorenstein point which is an index 2 type cA singularity.

Proof. Fix notation as in the proof of Theorem 1.6.

$P \in X$ is an isolated singularity and therefore by [Art69], [Art70] it is algebraic. Hence we can work in the algebraic category. Moreover we can assume that $X$ is Q-factorial.

Case 1. Assume that $\Gamma$ intersects one edge of the dual graph. Now proceed as in the proof of Theorem 1.6. From Proposition 4.5 it follows that $d=1$ and therefore $f^{-1}(\Gamma)=E_{1}+E_{2} . E_{1}, E_{2}$ are both smooth and hence $Y=B_{\Gamma} X$ is $c A_{*}$ along $L=E_{1} \cap E_{2}$. Moreover, $Y$ is smooth away from $L$. Let $C \subset Z$ be a $g$-exceptional curve. It must lie over a cDV point and therefore $Z$ can have at most finitely many terminal singularities along $C$. We now run a $\left(Z, S^{Z}+\epsilon E_{2}^{Z}\right)$ MMP. Observe that for any $g$-exceptional curve $C, C \cdot E_{2}^{Z}>0$ and therefore it cannot contract. Any other $f \circ g$-exceptional curve is $K_{Z}$-negative. So the first step of the MMP is the contraction of a $K_{Z}$ negative extremal ray. Moreover, since $\rho(Z / X)=2$, we play a 2 ray game and therefore the outcome of the $\left(Z, S^{Z}+\epsilon E_{2}^{Z}\right)$ MMP is the same as the outcome of the $(Z, 0)$ MMP. But now by [Ko-Mo98, Proposition 3.38] it is clear that $W$ is terminal. 
In order to study the index of the singularities of $W$ it is preferable to work in the analytic category. So we assume that $f: W \supset C \longrightarrow X \ni P$ is an extremal neighborhood. Let $C=\bigcup_{i=1}^{t}=f^{-1}(P)$ be the central curve. By [Kaw88, Lemma 3.4] it is possible to contract each one of the components of the central curve. Hence there is a factorization

$$
W \stackrel{g_{i}}{\longrightarrow} W_{i} \stackrel{h_{i}}{\longrightarrow} X
$$

where $g_{i}$ contracts $C_{i}$ and $f=h_{i} \circ g_{i}$. Moreover, since $S \in\left|-K_{X}\right|$ is $A_{n}, S_{W} \in\left|-K_{W}\right|$ is also $A_{m}$ for a $m \leqslant n$ and therefore $S_{W_{i}} \in\left|-K_{W_{i}}\right|$ is also $A_{r}$ (it dominates $S$ ). Apply [Ko-Mo92, Theorem 2.2] to $g_{i}$ to get that

$$
2 \leqslant \operatorname{index}(W) \leqslant n+1 .
$$

Now assume that the central curve $C$ is irreducible.

1. (b) follows from [Ko-Mo92, Theorem 2.2].

If this is not the case, then by the same theorem it follows that $W$ has exactly one non Gorenstein point which is of type $I A, I A^{\vee}$ or $I I A$, and $S^{W} \cong S$. In particular this implies that there are no flipping contractions during the $\left(Z, S^{Z}+\epsilon E_{2}^{Z}\right)$ MMP. Indeed, suppose there are. In the notation of the proof of Theorem 1.6, let $C \subset Z^{\prime}$ be one of the flipped curves. Then since this MMP is also the $(Z, 0)$ MMP, $K_{Z^{\prime}} \cdot C>0$. On the other hand, $K_{Z^{\prime}}+S^{Z^{\prime}}$ is crepant over $X$ and therefore $S^{Z^{\prime}} \cdot C<0$, which means that $C \subset S^{Z^{\prime}} . C$ is not contracted by $g^{\prime}$ and hence $S^{W}$ contains an exceptional curve which is impossible. Hence $Z^{\prime}=Z$. For convenience set $q=g^{\prime}$.

Now to find the index of $W$. Let $b \in \mathbb{N}$ such that $K_{Z}=q^{*} K_{W}+b E_{2}^{Z}$.

Claim. $E_{2}^{Z}$ has index $n$.

Let $S^{\prime}=f^{*} S$. Then $S^{\prime}$ has exactly 1 singular point which is $A_{n-1}$. This follows since $\Gamma^{\prime \prime}$ intersects the edge of the dual graph. So at the generic point of $L, Y$ has a $A_{n-1}$ point as follows from Lemma 4.4 and Proposition 4.5. At this point, $E_{1}, E_{2}$ correspond to two lines at the edge of the dual graph Therefore $n E_{1}, n E_{2}$ are Cartier at all but finitely many points.

Since $E_{1}+E_{2}$ is Cartier, it follows that the singularities of $Y$ lie on $L=E_{1} \cap E_{2}$. In fact if $n \geqslant 2$, then $Y$ is singular along $L$. If not then by Lemma 4.4, there is a $\Gamma \subset S \subset X$ such that $S^{\prime}=B_{\Gamma} S$ is smooth along $S^{\prime} \cap E_{1}$. But by Proposition 4.5 this implies that $S$ must be $A_{1}$ which is not possible.

Moreover, since $Z$ has has hypersurface singularities and therefore by Lemma 2.2 the indices of $E_{1}^{Z}$ and $E_{2}^{Z}$ can be computed at any point of $L^{Z}$. Therefore, they have index $n$.

Let $l \subset E_{2}^{Z}$ be the birational trasform of a line in $E_{2}=\mathbb{P}^{2}$ contracted by $p$. Then, $l \cdot E_{2}^{Z}=-a / n, a \in \mathbb{N}$. Moreover, $l \cdot E_{2}^{Z}+l \cdot E_{1}^{Z}=-1$, and clearly $l \cdot E_{1}^{Z} \leqslant 1$. Hence $-2 \leqslant l \cdot E_{2}^{Z} \leqslant-1$, and therefore $n \leqslant a \leqslant 2 n$. Moreover, $K_{Z} \cdot l=K_{Y} \cdot l=-1$ and hence $b=n / a$. In fact $a=n$ is not possible. If it was, then $K_{Z}=q^{*} K_{W}+E_{2}^{Z}$. But,

$$
K_{W}=p^{*} K_{X}+E_{1}^{W} \quad \text { and } \quad K_{Z}=h^{*} K_{X}+E_{1}^{Z}+E_{2}^{Z}
$$


where $h=f \circ g: Z \longrightarrow X$. Combining the above relations it follows that $q^{*} E_{1}^{W} \cong E_{1}^{Z}$, which is not possible. Therefore index $(W) \geqslant n+1$. But we already know that index $(W) \leqslant n+1$ and hence it must be equal. Moreover, from [Ko-Mo92, Theorem $2.2]$ it follows that the non Gorenstein point must have axial multiplicity 1 and hence it is cyclic quotient.

Case 2. $n=3$. The only case to study is when $\Gamma^{\prime \prime}$ intersects the middle of the dual graph. It will be necessary to obtain a normal form for the equation $\Gamma \subset S \subset X$.

LEMMA 5.2. Let $0 \in \Gamma \subset S \subset X$. Suppose that $0 \in S$ is an $A_{3}$ singular point and that $\Gamma^{\prime \prime}$ intersects the middle of the dual graph in the minimal resolution of $S$. Then under suitable coordinates, $0 \in \Gamma \subset S \subset X$ can be written as

$$
x^{2}+y^{2}+2 x z^{2}+t \phi_{\geqslant 1}(x, z, t)=0
$$

and $I_{\Gamma}=(x, y, t)$ and no power $z^{k}$ appears in $\phi_{\geqslant 1}(x, z, t)$.

Proof. By Theorem 4.1, under suitable coordinates it is possible to write $S: x y-z^{5}=0$ and $\Gamma: x-z^{2}=y-z^{2}=0$. The change of coordinates $x \mapsto x+z^{2}$, $y \mapsto y+z^{2}$ brings it to $x y+x z^{2}+y z^{2}=0$ and $\Gamma: x=y=0$. Now let $x \mapsto x-y$, $y \mapsto x+y$ and apply the Weierstrass preparation theorem to $y^{2}$ to get

$$
S: x^{2}+y^{2}+2 x z^{2}+t \phi_{\geqslant 1}(x, z, t)=0
$$

and $\Gamma: x=y=t=0$. To eliminate $z^{k}$ we must show that $z$ does not appear in $\phi_{\geqslant 1}(x, z, t)$. If it does then it is easy to see that the general hyperplane section $y=a t+b x$ through $\Gamma$ will be $A_{1}$ which is impossible. Now eliminate the powers of $z$ as in Proposition 4.7.

The statement about existence of a terminal contraction is proved in exactly the same way as Theorem 6.1 and I omit its proof.

EXAMPLE 5.3. Let $X$ be given by

$$
x^{2}+y^{2}+2 x z^{2}+t^{m}=0
$$

and $\Gamma: x=y=t=0$. Then if $m \geqslant 4$, there is no 3-fold terminal contraction, contracting a surface to $\Gamma$.

Proof. In this case, the section $S:(t=0)$ is an $A_{3}$ type and the curve intersects the middle of the minimal resolution of $S$. Moreover, $f_{\leqslant 3}(x, z, t)=2 x z^{2}$ is reducible and therefore by the previous theorem there is no terminal contraction.

\section{The $D_{2 n}$ Cases}

In this section we will study the existence of terminal contractions when $0 \in S$ is a $D_{2 n}$ type for general $0 \in \Gamma \subset S \subset X$. 
THEOREM 6.1. Let $P \in \Gamma \subset S \subset X$. Suppose that $P \in S$ is a $D_{n}$ type singular point. Then

(1) Suppose that $\Gamma \subset S$ is of type $F D_{l}$. If $n \geqslant 5$, then there is no terminal contraction. If $n=4$ then the next case holds.

(2) Suppose that $\Gamma \subset S$ is of type $F D_{r}$ and $n$ is even. Then write the equation of $P \in \Gamma \subset S \subset X$

$$
x^{2}+f_{3}(y, z, t)+f_{\geqslant 4}(y, z, t)=0
$$

with $I_{\Gamma}=(x, y, t)$.

(a) If $f_{3}(y, z, t)$ is an irreducible homogeneous cubic, then there is a terminal contraction $W \longrightarrow X$ of a surface to $\Gamma$. W has index 2 and has exactly one singularity which is of $c D$ type. Moreover, $R(\Gamma, X)=\bigoplus_{d \geqslant 0} \mathcal{I}_{\Gamma, X}^{(d)}$ is finitely generated by elements of degrees 1 and 2 .

(b) If $f_{3}(y, z, t)$ is reducible or 0 , then there is no terminal contraction.

Proof. We will only do the second part of the theorem. The first one is proved in exactly the same way.

By Proposition 4.7, under suitable coordinates $\Gamma \subset S \subset X$ is given by

$$
x^{2}+y^{2} z+2 y z^{m}+t \phi_{\geqslant 2}(y, z, t)=0,
$$

$\Gamma: x=y=t=0$ and no power $z^{k}$ appears. Let $f: Y \longrightarrow X$ be the blow up of $\Gamma$. In the chart $x=x t, y=y t, Y$ is given by

$$
x^{2} t+y^{2} z t+2 y z^{m}+\phi_{\geqslant 2}(y t, z, t)=0 .
$$

For $t=0$ we find that $f^{-1}(\Gamma)=E_{1}+m E_{2}$, and $E_{1}: y=t=0, E_{2}: z=t=0$. It is easy to check that $Y$ has exactly one singular point on $L=E_{1} \cap E_{2}=(y, z, t)$. Therefore, $g: Z=B_{E_{1}} Y \longrightarrow Y$ is the Q-factorialization of $E_{1}$ (and hence $E_{2}$ ). Moreover, $E_{2}^{Z} \cong E_{2}=\mathbb{P}^{2}$. Now as in the proof of Theorem 1.6 contract $E_{2}^{Z}$ to get a morphism $Z \stackrel{\vec{p}}{\longrightarrow} W$ over $X$. Then $W \stackrel{q}{\longrightarrow} X$ is the required contraction. Whether or not $W$ is terminal depends on what kind of singularities $Z$ has away from $E_{2}^{Z}$. Let

$$
\phi_{\geqslant 2}(y, z, t)=\phi_{2}(y, z, t)+\phi_{\geqslant 3}(y, z, t) .
$$

In the chart $y=y t, Z$ is given by

$$
x^{2}+y^{2} t^{2} z+2 y z^{m}+\frac{1}{t} \phi_{2}\left(y t^{2}, z, t\right)+\frac{1}{t} \phi_{\geqslant 3}\left(y t^{2}, z, t\right)=0 .
$$

Write

$$
\phi_{\geqslant 3}\left(y t^{2}, z, t\right)=t f_{\geqslant 2}(z)+t^{2} f_{\geqslant 1}(y, z, t)
$$

with $f_{\geqslant 1}(y, 0,0)=0$. Then $Z$ is given by

$$
F=x^{2}+y^{2} t^{2} z+2 y z^{m}+\frac{1}{t} \phi_{2}\left(y t^{2}, z, t\right)+f_{\geqslant 2}(z)+t f_{\geqslant 1}(y, z, t)=0 .
$$


Let $C=g^{-1}(0): x=z=t=0$, and

$$
\Phi_{2}(y, z, t)=\frac{1}{t} \phi_{2}\left(y t^{2}, z, t\right) .
$$

We want to investigate the singularities of $Z$ along $C$.

$$
\begin{aligned}
& \frac{\partial F}{\partial x}=2 x, \\
& \frac{\partial F}{\partial y}=2 y z t^{2}+2 z^{m}+\frac{\partial \Phi_{2}}{\partial y}+t \frac{\partial f_{\geqslant 1}}{\partial y}, \\
& \frac{\partial F}{\partial z}=y^{2} t^{2}+2 m y z^{m-1}+\frac{\partial \Phi_{2}}{\partial z}+\frac{\partial f_{\geqslant 2}}{\partial z}+t \frac{\partial f_{\geqslant 1}}{\partial z}, \\
& \frac{\partial F}{\partial t}=2 y^{2} t z+\frac{\partial \Phi_{2}}{\partial t}+f_{\geqslant 1}(y, z, t)+t \frac{\partial f_{\geqslant 1}}{\partial t} .
\end{aligned}
$$

Hence $Z$ is singular along a point $Q \in C$ iff

$$
\frac{\partial \Phi_{2}}{\partial y}(Q)=\frac{\partial \Phi_{2}}{\partial z}(Q)=\frac{\partial \Phi_{2}}{\partial t}(Q)=0 .
$$

Let $\phi_{2}(y, z, t)=a_{1} y^{2}+a_{2} t^{2}+a_{3} y t+a_{4} y z+a_{5} z t$. Then

$$
\Phi_{2}(y, z, t)=\frac{1}{t} \phi_{2}\left(y t^{2}, z, t\right)=a_{1} y^{2} t^{3}+a_{2} t+a_{3} y t^{2}+a_{4} y z t+a_{5} z .
$$

Now it follows that

$$
\begin{aligned}
& \frac{\partial \Phi_{2}}{\partial y}=2 a_{1} y t^{3}+a_{3} t^{2}+a_{4} z t, \\
& \frac{\partial \Phi_{2}}{\partial z}=a_{4} y t+a_{5}, \\
& \frac{\partial \Phi_{2}}{\partial t}=3 a_{1} y^{2} t^{2}+a_{2}+2 a_{3} y t+a_{4} y z .
\end{aligned}
$$

Along $C$,

$$
\frac{\partial \Phi_{2}}{\partial y}=0, \quad \frac{\partial \Phi_{2}}{\partial z}=a_{5}, \quad \frac{\partial \Phi_{2}}{\partial t}=a_{2} .
$$

Therefore, $Z$ is either singular along $C$ and $a_{2}=a_{5}=0$, or has exactly one singular point in $E_{2}^{Z}$ (in the other chart). If $a_{2}=a_{5}=0$, then

$$
\phi_{2}(y, z, t)=y\left(a_{1} y+a_{3} t+a_{4} z\right) .
$$

A coordinate independent way to say this is the statement of Theorem 6.1(2b).

We will now find the index of the singularities of $W$. Let $a>0$ such that $K_{Z}=p^{*} K_{W}+a E_{2}^{Z}$. Moreover, $E_{2}^{Z} \cong E_{2} \cong \mathrm{P}^{2} . E_{1}^{Z}$ is Cartier and therefore for a general line $l \subset E_{2}^{Z}, l \cdot E_{1}^{Z}=1$. On the other hand,

$$
l \cdot\left(E_{1}^{Z}+2 E_{2}^{Z}\right)=l \cdot g^{*}\left(E_{1}+2 E_{2}\right)=l \cdot\left(E_{1}+2 E_{2}\right)=-1 .
$$


Hence $l \cdot E_{2}^{Z}=-1$. Moreover,

$$
l \cdot K_{Z}=l \cdot g^{*} K_{Y}=l \cdot K_{Y}=-1 .
$$

Combining the above we see that $a=1$ and therefore $K_{Z}=p^{*} K_{W}+E_{2}^{Z} \cdot E_{2}^{Z}$ has index 2 and hence $W$ has also index 2. From the above proof it is also clear that $W$ has exactly one index 2 point. This, as well as the type of the singularities, also follows from [Ko-Mo92, Theorem 4.7].

Moreover, since $W$ has index 2 it follows that $-2 E$ if $p$-very ample where $E=E_{1}^{W}$. The statement about the number of generators of $R(\Gamma, X)$ follows immediately.

The difference between the $D_{2 n}$ and $D_{2 m+1}$ cases is shown by the next lemma.

LEMMA 6.2. Suppose that the general section $S$ of $X$ containing $\Gamma$ is $D_{2 n+1}$ and $\Gamma \subset S$ is of type DF . Let $f: Y=B_{\Gamma} X \longrightarrow X$. Let $f^{-1}(\Gamma)=E_{1}+\mathrm{d} E_{2}$. Then $Y$ is singular along $L=E_{1} \cap E_{2}$.

This is precisely the reason that makes the $D_{2 n+1}$ case very difficult to work with. In the $D_{2 n}$ cases, $Y$ had exactly one singular point on $L$ and that made an explicit description of the Q-factorialization of $E_{1}$ relatively easy.

Proof. Suppose that $Y$ is not singular along $L$. Let $Q \in L$ be a smooth point. Let $S^{\prime}$ be a general section of $Y$ through $Q$. As in Lemma 4.4, there is a section $S$ of $X$ through $\Gamma$ such that $S^{\prime}=f_{*}^{-1} S$. Then by assumption, $S$ is $D_{2 n+1}$. Then by Proposition 4.5.2.b(ii), it follows that $Q \in S^{\prime}$ is singular which is not true.

To apply Theorem 6.1, it would be useful to get information about the general section from a special section. The next lemma gives informaion about the general section starting from a special one.

LEMMA 6.3. Let $\Gamma \subset X$. Suppose that the general section of $X$ containing $\Gamma$ is $D_{k}$. Let $P \in \Gamma \subset S_{0} \subset X$ be a special section and suppose that $S_{0}$ is $D_{n}$ with $n \geqslant 5$. Then

(1) If $\Gamma \subset S_{0}$ is of type $D F_{l}$, then the general section $S$ of $X$ through $\Gamma$ is $D_{m}, m \leqslant n$ and also of type $D F_{l}$.

(2) If $\Gamma \subset S_{0}$ is of type $D F_{r}$ and $n$ is even, then the general $S$ through $\Gamma$ is $D_{2 k}$ and also of type $D F_{r}$.

Proof. Case 1. Suppose that $\Gamma \subset S_{0}$ is of type $F D_{l}$, i.e., $\Gamma^{\prime \prime}$ intersects $E_{1}$ in the fundamental cycle of $S_{0}$. Let $S$ be the general section through $\Gamma$ and assume it is $D_{m}$. If $\Gamma^{\prime \prime}$ intersects $E_{m-1}$ or $E_{m}$ in the fundamental cycle of $S$, then by Proposition 4.5 it follows that $d=m / 2$, if $m$ is even, or $d=(m-1) / 2$, if $m$ is odd. On the other hand, by 
the assumption on $S_{0}$ and Proposition 4.7, under suitable coordinates $\Gamma \subset X$ is given by $x^{2}+y^{2} z+z^{n-1}+t \phi_{\geqslant 2}(y, z, t)=0$, and $I_{\Gamma}=(x, z, t)$. Use notation as in Lemma 6.2.

A computation as in Theorem 6.1 shows that $d=2$ and $Y$ has exactly one singular point on $L$. Hence, the only possibility that the general section is not as claimed is that it is $D_{5}$ and $\Gamma^{\prime \prime}$ intersects $E_{4}$ or $E_{5}$. But then $Y$ is singular along $L$ as follows from Lemma 6.2.

The fact that $m \leqslant n$ follows from the upper semicontinuity of the Tyurina number of the singularity.

Case 2. Suppose that $\Gamma \subset S_{0}$ is of type $F D_{r}$ and $n$ is even. First we will show that it is not possible that $\Gamma \subset S, S \subset X$ general through $\Gamma$, is of type $F D_{l}$. Suppose it is. Then since $n$ is even, $S^{\prime}=f_{*}^{-1} S$ has exactly one singular point which is $D_{n-1}$ as follows from Proposition 4.6.2.a. On the other hand, $S_{0}^{\prime}$ has exactly one $A_{n-1}$ singular point $Q$. Therefore $Q \in Y$ is $c A_{k}$ and by [KoBa88] it is $c A_{k}$ in a neighborhood of $Q$. But then for a general $\Gamma \subset S \subset X, S^{\prime}$ is $A_{k}$ and hence it must be of type $F D_{r}$. If $\Gamma \subset S$ is $F D_{r}$ but $S$ is $D_{2 m+1}$ for general $S$, then by Lemma $6.2 Y$ is singular along $L$ which is not true as follows from Corollary 4.6.

Hence by looking at one section we know in which part of Theorem 6.1 we are. So if we know that there is a section as in 1 then all we need to know to conclude that there is no terminal contraction is that the general section is not $D_{4}$. The next lemma gives a criterion for that.

LEMMA 6.4. Let $\Gamma \subset X$ be given by $x^{2}+f_{\geqslant 3}(y, z, t)=0$, and $\Gamma=(x, y, t)$. Moreover, suppose that $t=0$ is a DuVal section $S$ of $X$ containing $\Gamma$ and $\Gamma^{\prime \prime}$ intersects $E_{1}$ in the fundamental cycle of $S$. Then a $D_{4}$ section of $X$ containing $\Gamma$ does not exist iff $f_{3}(y, z, t)=g(y, z, t) h^{2}(y, z, t)$.

Proof. According to Proposition 4.7, in suitable coordinates $\Gamma \subset X$ is given by

$$
x^{2}+y^{2} z+z^{n-1}+t \phi_{\geqslant 2}(y, z, t)=0,
$$

and $I_{\Gamma}=(x, z, t)$. The cubic term then of the above equation is

$$
q_{3}(y, z, t)=y^{2} z+t \phi_{2}(y, z, t) .
$$

From the proof of the first part of Proposition 4.7 it follows that a $D_{4}$ section exists iff $q_{3}(y, z, t) \neq g(y, z, t) h^{2}(y, z, t)$, for any $g(y, z, t), h(y, z, t)$, which is the condition claimed by the Lemma.

\section{Acknowledgements}

The author is supported by the Marie Curie research fellowship grant number MCFI-1999-00448.

Part of this work was done during my visit to Princeton University in April 2001 and I would like to thank János Kollár for many interesting and useful discussions while at Princeton, and Miles Reid who suggested this problem to me. 


\section{References}

[Art66] Artin, M.: On isolated rational singularities of surfaces, Amer. J. Math. 88 (1966), 129-136.

[Art69] Artin, M.: Algebraic approximation of structures over complete local rings, Publ. Math. IHES 36 (1969), 23-58.

[Art70] Artin, M.: Algebraisation of formal moduli II, Ann. of Math. 91 (1970), 88-135.

[Benv85] Benveniste, X.: Sur le cone des 1-cycles effectifs en dimension 3, Math. Ann. 272 (1985), 257-265.

[Cor95] Corti, A.: Factoring birational maps of threefolds after Sarkisov, J. Algebraic Geom. 4 (1995), 223-254.

[Cor-Rei00] Corti, A. and Reid, M.: Explicit Birational Geometry of 3-folds, Lecture Notes in Math. 281, Springer, New York, 2000.

[Cut88a] Cutkosky, S.: Elementary contractions of Gorenstein threefolds, Math. Ann. 280 (1988), 521-525.

[Cut88b] Cutkosky, S.: Weil divisors and symbolic algebras, Duke Math. J. 57 (1988), 175-183.

[Jaf92] Jaffe, D. B.: Local geometry of smooth curves passing through rational double points, Math. Ann. 294 (1992), 645-660.

[ka01] Kawakita, M.: Divisorial contractions in dimension three which contract divisors to smooth points. Invent. Math. 145(1) (2001), 105-119.

[Kaw88] Kawamata, Y.: Crepant blowing up of 3-dimensional canonical singularities and its application to degenerations of surfaces, Ann. of Math. 127 (1988), 93-163.

[Kaw94] Kawamata, Y.: Divisorial contractions to 3-dimensional terminal quotient singularities, In: Higher-Dimensional Complex Varieties (Trento 1994), de Gruyter, Berlin, (1996), pp. 241-246.

[Ko97] Kollár, J.: Singularities of pairs, Proc. Sympos. Pure Math. Soc. 62 (1997).

[Ko91] Kollár, J.: Flips, flops, minimal models, etc., Surveys in Differential Geom. 1 (1992), 293-323.

[KoBa88] Kollár, J. and Shepherd-Barron, N. I.: Threefolds and deformations of surface singularities, Invent. Math. 91 (1988), 299-338.

[Ko-Mo98] Kollár, J. and Mori, S.: Birational Geometry of Algebraic Varieties, Cambridge University Press, 1988.

[Ko-Mo92] Kollár, J. and Mori, S.: Classification of three-dimensional flips, J. Amer. Math. Soc. 5(3) (1992), 533-703.

[Mil68] Milnor, J.: Singular Points of Complex Hypersurfaces, Princeton University Press, 1968.

[Rei87] Reid, M.: Young person's guide to canonical singularities, Proc. Sympos. Pure Math. Soc. 46 (1987). 